\title{
Experimental Observations of the Visibility Threshold of Illusory effects in Hermann Grid, Sinusoidal and Square Gratings and their Possible Implications
}

\author{
Ashish Bakshi ${ }^{1}$, Sourya Roy ${ }^{2}$, Arijit Mallick ${ }^{3}$ and Kuntal Ghosh ${ }^{14^{*}}$ \\ ${ }^{1}$ Machine Intelligence Unit, Indian Statistical Institute, Kolkata \\ ${ }^{2}$ Department of Electronics and Instrumentation, Jadavpur University, Kolkata \\ ${ }^{3}$ IRCCYN, Ecole Centrale de Nantes, France \\ ${ }^{4}$ Center for Soft Computing Research, Indian Statistical Institute, Kolkata
}

Received: 19 May, 2017; Accepted: 08 June, 2017 ; Published: 19 June, 2017

*Corresponding author: Kuntal Ghosh, Machine Intelligence Unit, Indian Statistical Institute, Kolkata, India; E-mail: kuntal@isical.ac.in

\begin{abstract}
Experimental studies have been conducted on the Sinusoidal Grating, Square Grating and Hermann Grid Illusions. Volunteers were presented with a range of input stimuli and asked to report the perceptibility of the illusory effect. We tried to find the thresholds for the parameters within which the illusion is visible. Some noteworthy observations were made from these experiments. We have observed that the length scale of the illusory effect changes with the typical length scale of the input stimulus, almost in proportion, wherefrom one can draw the conclusion that most spatial filters, possessing typical length scales of their own, would not respond to stimuli much smaller or much larger than that typical length scale. We have also found that even tiny changes to the pattern of the Hermann grid illusion is sufficient to wipe out the illusion completely, i.e. the illusion is very sensitive to tiny changes in the input stimulus. These observations indicate that simple linear models such as the DOG (Difference of Gaussians) model or even the highly successful ODOG (Oriented Difference of Gaussians) model of brightness perception are actually inadequate for explaining such effects. The present work thus bears implication for future efforts towards modeling the human visual system, by pointing out certain features that such models must exhibit.
\end{abstract}

Keywords: Brightness illusion; Perceptible threshold; Scaling effect

\section{Introduction}

The visual system perceives the surroundings in its own complex way. This complex structure of the visual system is sometimes exposed by its illusions [1]. In presence of physical stimuli (optical), excitations and sensations are generated in the brain. By analyzing these phenomena, we can explore the properties of the visual sensory system. For this purpose, various psychophysical methods and experiments are widely used. Psychophysics requires objectification of the subjective response [2]. This task can involve 'detection', 'identification' or 'discrimination' of various stimuli. In his publication, Elemente der Psychophysik, Gustav Theodor Fechner (1801-1887) first introduced a class of techniques and psychophysical methods in order to correlate stimulus with sensation [2,3]. Many variations and extensions of these classical methods have since then been proposed, but the very essence of psychophysical experimentations remained same i.e., measurement, characterization and quantification of the perceptual experience using some psychophysical quantities. According to Fechner, and in fact the same was indicated even before him by Johann Friedrich Herbart (1776-1841), Absolute Threshold of any stimulus intensity is an important measurable quantity to judge any stimulus and objectify it's perception in the brain. This was the primary motivation behind the selection of 'absolute threshold' as the focus of this work. The term 'absolute threshold' is defined as the minimum intensity level of stimulus that can be detected reliably by any subject. In this work we have performed some experimental studies on brightness contrast illusions to find the absolute thresholds of perceptibility of the illusory effects. These thresholds were measured as a function of certain characteristic parameters of the stimuli. We have worked on Brightnesscontrast illusions which are considered very important because a complete understanding of these illusions can help us get an insight into the physiological mechanism of lateral inhibition and the cognitive mechanism of brightness perception in our brain. In this particular work, we have taken two widely known brightness contrast illusions the Hermann grid illusion and the Grating induction, for experimentation.

The Hermann grid illusion was first reported by Ludimar Hermann in 1870. The Hermann grid illusion consists of gray illusory blobs perceived at the intersections of horizontal and vertical white strips viewed against a dark background. When we concentrate on one single intersection, the blob disappears from that particular intersection but can be seen at all other crossings. Several works in literature bear testimony to the importance of studying the Hermann grid and other brightness- 
contrast illusions towards a better understanding of receptive field organizations, lateral inhibition phenomenon, the parallel channels in visual pathway and so on [4,5]. Davies and Morland have demonstrated the disruption of lateral inhibition processing in diabetes mellitus by designing experiments to measure the threshold of perception of the Hermann grid illusion for different bar widths, a topic that we have dealt elaborately in this paper especially in Experiment 3 [6]. It is to be noted that two of the three experiments dealt in this paper concerns the Hermann grid illusion as its importance is being emphasized again and again in the performance of various visual tasks. Gilchrist et al., for instance, have used it as a tool in making estimates of contrast perception for dyslexics and non-dyslexics [7].

The grating induction illusion, on the other hand, occurs when a strip of homogenous luminosity is placed in the middle of a sine-wave grating, the strip is not perceived as a uniform one, but it also looks like a grating. This visual effect is called grating induction illusion and was first shown by McCourt [8]. The square-wave grating in place of sine-wave also produces a similar effect. Both of these variants of grating induction illusion have been used in our work. For all the discussions henceforth, the 'homogeneous strip' will be termed as 'test strip' or 'test patch'.

Our experiments relating to the above mentioned illusions have been described systematically in the sections below. The results from our present study have been discussed individually with associated experiments and a section with assimilated discussions and results from all the experiments has also been presented.

\section{General Methodology and Apparatus}

All stimuli were generated on a PC with a 17" LCD display at $1280 \times 1024$ resolutions. Frame refresh rate was 60 Hz. Color depth was 24 bit ( 8 bits each for R, G, B channels). The display unit was calibrated using a photometer and the calibration data was used to linearise the display. All stimuli were shown to the subjects against a background with grayscale intensity 128 . Distance between screen and observer was fixed at $70 \mathrm{~cm}$. The subjects who participated in our experiments are among the authors and are all well-trained in psychophysical experiments. All stimuli were created and shown using the Psychophysics toolbox of Matlab.

We used the psychophysical method of limits which is a classical method proposed by Fechner for the first time. We started first by showing the subject a particular illusion and then asking whether the illusory effect is perceivable or not. For every stimulus shown, the subjects responded with either YES or NO depending upon whether the illusory effect was visible or not. Then we started to change specific parameters of interest, related to that illusion in successive and discrete steps and at each step we checked the perceptibility. Values of the parameters at which the transition from YES to NO (or from NO to YES) occurs were marked and from these transition values the absolute thresholds for those parameters were calculated. The experiments were repeated 10 times for each parameter and the average value of the thresholds was taken as the final value of absolute threshold for that specific parameter.

\section{Experiment 1}

\section{Stimuli}

In this experiment a modified version of Hermann grid illusion was used as the stimulus as shown in Figure 1a-c. The grid shown to our subjects contained 81 black squares in it. The separation between the squares was 0.01 radians. This value of separation and the range of the square sizes were selected using the observations of the Experiment 3 described in this paper, so that the subjects could perceive the illusions most prominently. In this particular experiment, the size of stimulus i.e. Hermann grid was not kept constant during the experiment.

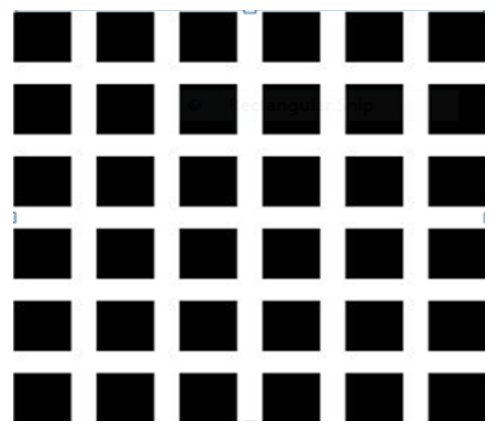

Figure 1a: Hermann grid stimulus. Illusory blobs are prominently visible at the intersections.

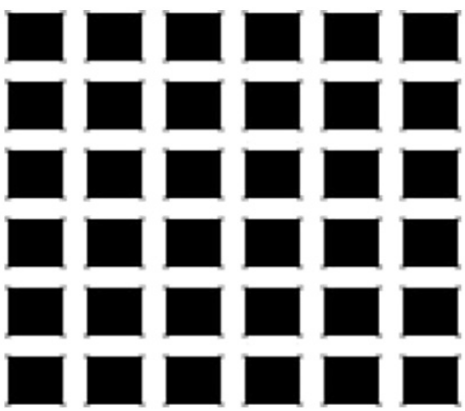

Figure 1b: Modified Hermann grid stimulus with tiny grey squares added to the corners. The illusory effect is reduced than that in Figure 1a.

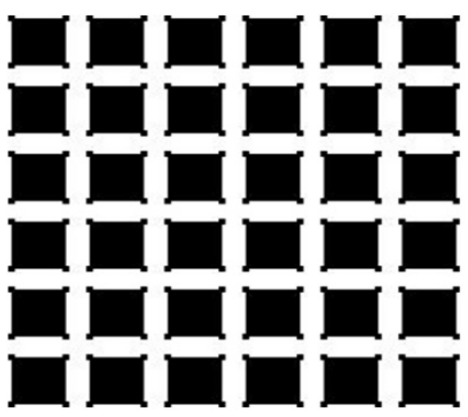

Figure 1c: Modified Hermann grid stimulus with tiny black squares added to the corners. No illusory blobs can be seen anymore.. 


\section{Procedure}

As mentioned earlier the subjects were shown a modified Hermann grid, in which tiny squares were added to all the four corners of the Hermann grid squares. The added squares constitute less than $2 \%$ of the area of the unmodified Hermann grid stimulus. A series of such stimuli were shown to the subjects with increasing width of the grid squares. Since the total number of squares was kept constant the size of the entire illusion was also changing accordingly. For each stimulus, we changed the gray-level intensity of the added squares in steps, from white to black, as shown in Figures 1a-1c and the subjects were asked if the illusion was visible or not. The time interval between two steps was kept same as in the previous experiment. As the graylevel intensities were varied, if any alteration in subject response occurred we noted that particular gray-level intensity and from this information, we later calculated the gray-level intensity threshold of those tiny squares for a Hermann grid with specific square width. This procedure was repeated for different choices of square width. The largest grid in our experiments had squares of 0.04 radians width and the smallest one had 0.03 radians.

\section{Observations and discussions}

The most interesting outcome of this experiment is that very little modification of the Hermann grid (like adding very tiny squares with less than $2 \%$ area of the unmodified stimulus) is sufficient to wipe out the illusory perception of the dark blobs. Usage of the phrase "very little modification" will be more apparent if we compare our amount of alterations in the Hermann grid with those in the existing literatures [9].

Gray-level intensity threshold vs. square side-length plots for the two subjects have been illustrated in Figure 2. From these graphs, it can be clearly observed that, for a particular subject, irrespective of the size of the original squares, there is a fixed gray-level intensity threshold of the added small squares, below which the illusion becomes imperceptible.

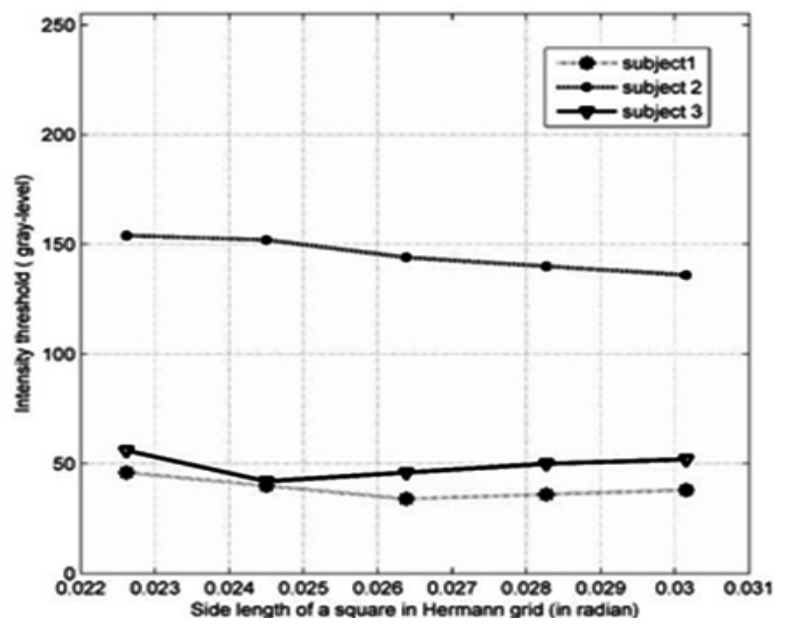

Figure 2: Intensity threshold vs. side length (of Hermann grid squares) graph.
The last point about the experiment which needed to be mentioned is that the size of the added squares does not need to be modified when the size of the stimulus is being changed. Exactly same number of added squares could remove the illusory visual effect from the smallest as well as the biggest version of Hermann grid stimulus.

\section{Experiment 2}

\section{Stimuli}

This experiment was done using the sine-wave grating and square-wave grating stimuli. Stimuli, such as shown in Figure 3a-3 h, were shown to the subjects at various cycle frequencies. Cycle frequencies ranged from 20 cycles/radian to 121 cycles/ radian in case of sine-wave grating. For the square-wave grating, minimum frequency was 20 cycles/radian and maximum

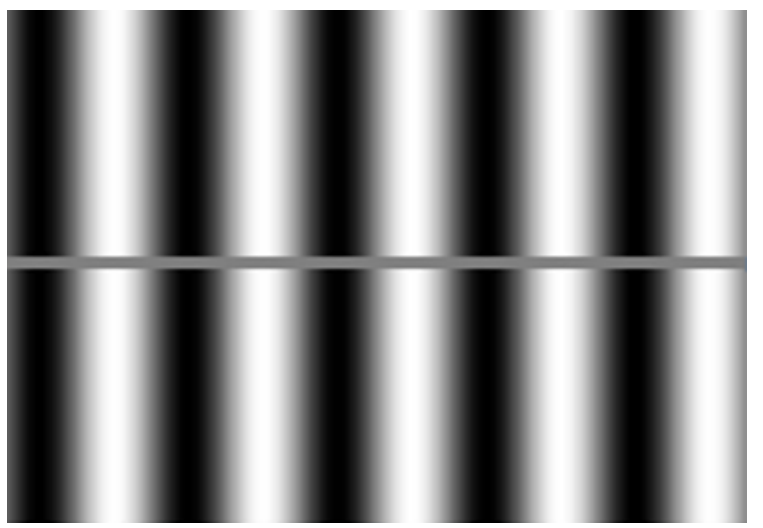

Figure 3a: Low frequency sine-wave grating stimulus with narrow gray strip. Illusory brightness undulations are visible all throughout the gray-strip.

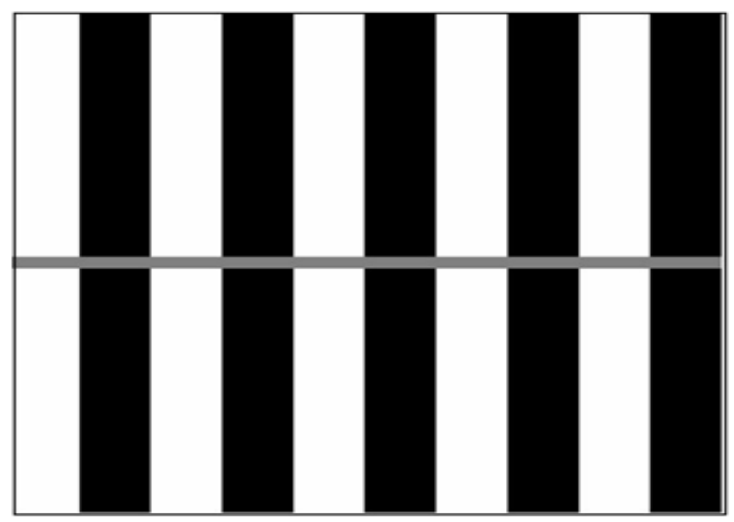

Figure 3b: Low frequency square-wave grating stimulus with narrow gray strip. Illusory brightness undulations are visible all throughout the gray-strip. 


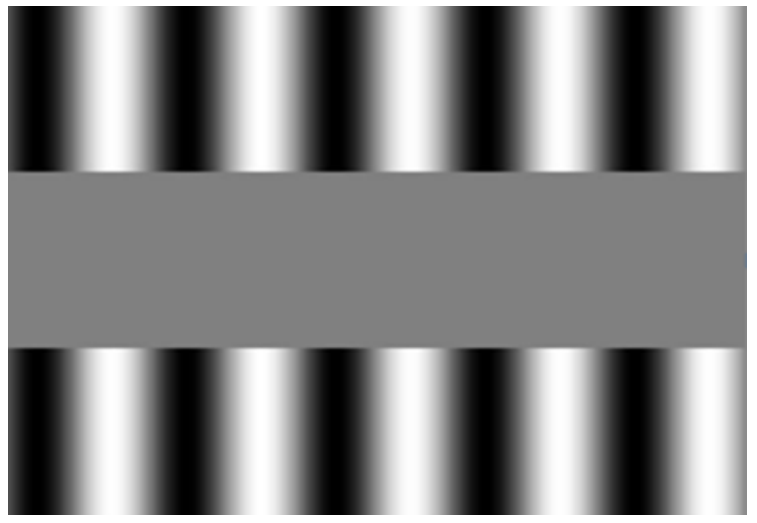

Figure 3c: Low frequency sine-wave grating stimulus with wide graystrip. Illusory brightness undulations are prominent only along the upper and lower edges of the gray-strip.

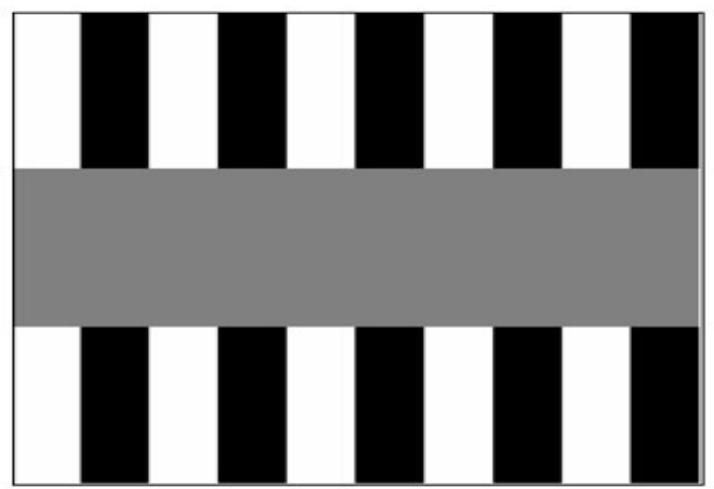

Figure 3d: Low frequency square-wave grating stimulus with wide gray-strip. Illusory brightness undulations are prominent only along the upper and lower edges of the gray-strip

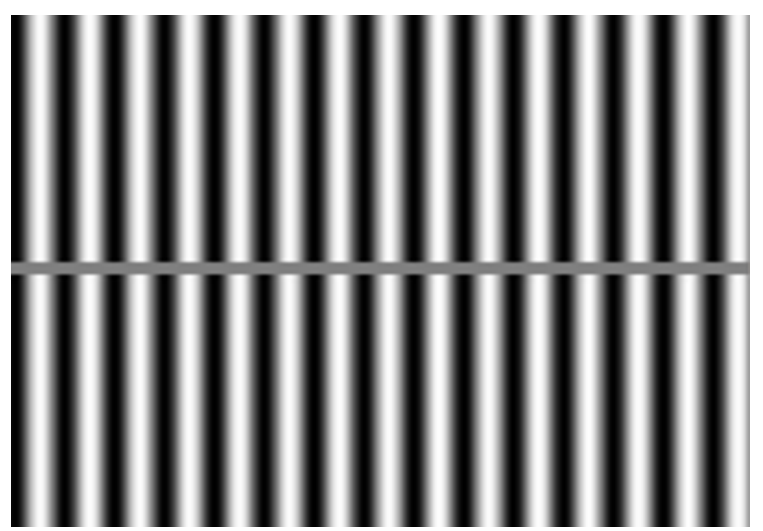

Figure 3e: High frequency sine-wave grating stimulus with narrow gray- strip. Illusory brightness undulations are visible all throughout the gray-strip.

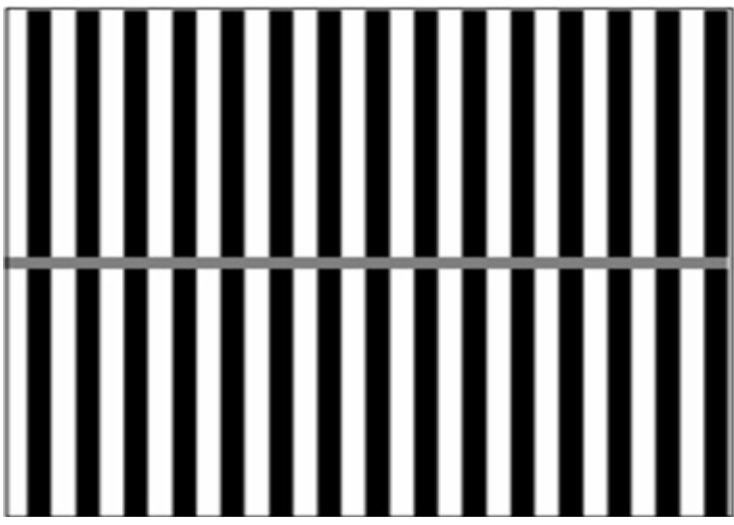

Figure 3f: High frequency square-wave grating stimulus with narrow gray- strip. Illusory brightness undulations are visible all throughout the gray-strip.

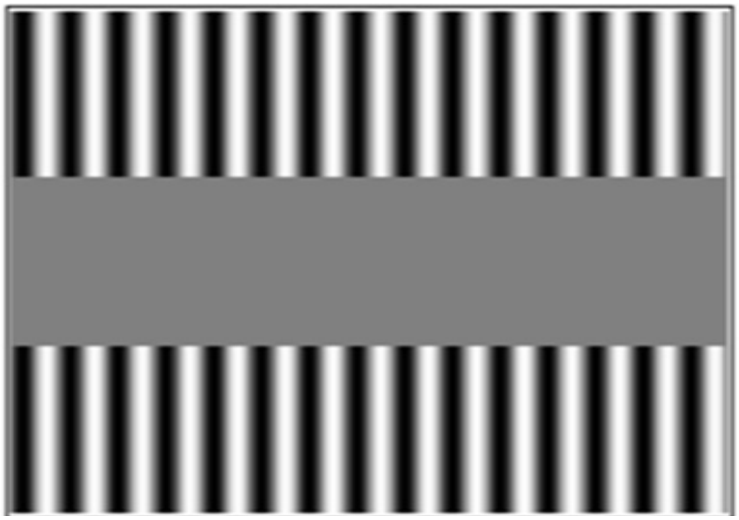

Figure 3g: High frequency sine-wave grating stimulus with wide gray strip. Illusory brightness undulations are prominent only along the upper and lower edges of the gray-strip.

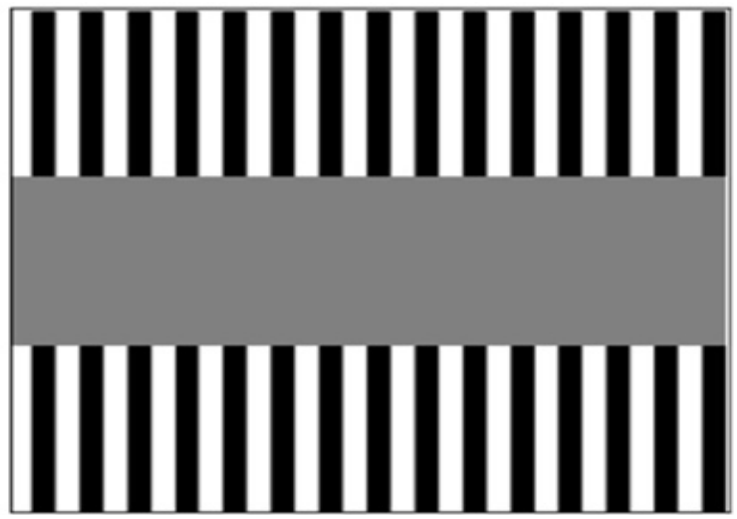

Figure 3h: High frequency square-wave grating stimulus with wide gray strip. Illusory brightness undulations are prominent only along the upper and lower edges of the gray-strip. 


\section{Procedure}

Before the experiments, the subjects were clearly instructed to concentrate on the approximate horizontal centerline of the strip and report about the perceptibility of the illusion, i.e. whether or not brightness undulations could be seen along the horizontal centerline of the gray-strip. For any specific value of cycle frequency of grating, we started with a width of 0.002 radians of the gray-strip. Then we increased the width in steps until the gray strip fully overlaid the grating and marked that strip width at which response of the subject reverses (response changes from 'yes' to 'no' or 'no' to 'yes'). And from this value of strip width, at which the transition in response occurred, the absolute threshold for strip width was determined for a particular frequency. This procedure was done for each spatial frequency of both sine-wave and square-wave grating. During the experiment the width of the strip was being changed using key presses. For each key press the width of the strip was being changed by 0.0005 radians. The interval between two successive key presses was approximately 10 seconds.

\section{Observations and discussions}

The results from the experiment 2 have been demonstrated in Figure 4a and 4b. Plot for each subject has been presented separately. From these figures it can be distinctly seen that for every subject and at each spatial frequency of the grating there exists a threshold width of the gray test strip above which the illusion is not visible i.e. if we increase the width of the test strip above this threshold value, the illusion becomes undetectable. Even though the values of thresholds at different frequencies can vary from subject to subject, some threshold must exist for each subject.

The results also show that as the spatial cycle-width of the grating increases the threshold also increases. Lastly, we can see that irrespective of the subject, at any given wavelength of the background waveform the threshold value for square-wave grating is smaller than that for sine-wave grating. This clearly indicates, in agreement with the previous assertions, that the sinewave grating induction illusion is much stronger than squarewave grating induction illusion [10] (Figure 4a).

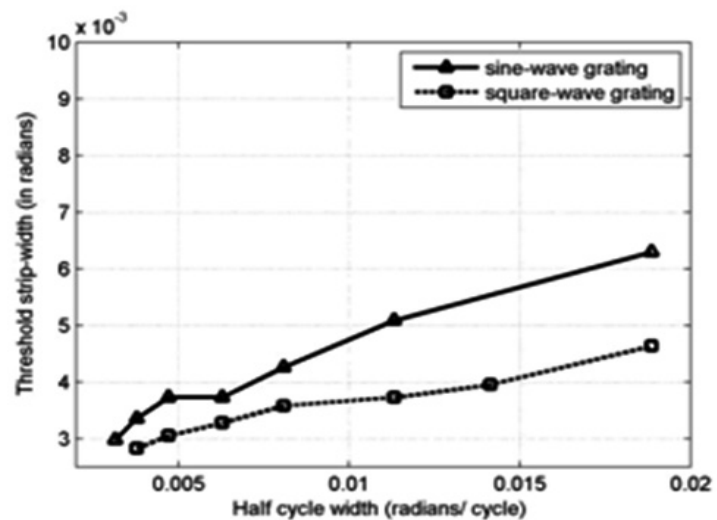

Figure 4a: Threshold strip-width vs. spatial frequency curve for subject 1.

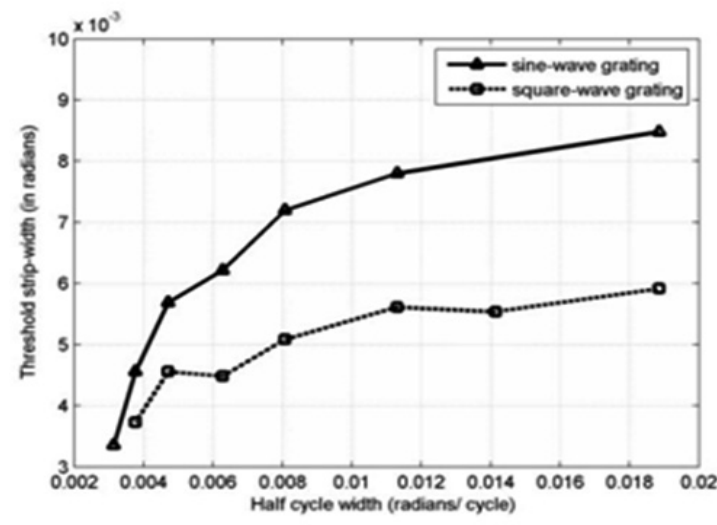

Figure 4b: Threshold strip-width vs. spatial frequency curve for subject 2.

\section{Experiment 3 \\ Stimuli}

The Hermann grid was again used as the stimulus in this illusion as shown in Figure 5a-5c. The subjects were shown several sequences of Hermann grid illusions which differed from one another by the size of their squares. Maximum side-length of squares was taken 0.03 radians and the minimum count was 0.008 radians. In this range we varied the side-length of the squares in the grid.

\section{Procedure}

For any subject, we started with a Hermann grid with specific square side-length and asked him whether he could see the illusion or not. Initially, the width of separation between the squares was taken to be $5 \times 10^{\wedge}(-4)$ radians. Then we increased the separation width between the squares, step-by-step, keeping the square size constant, as shown in Figure $5 a-5 c$, while recording the perceptibility of the illusion by the subject. At each step, if any transition in subject response occurred we noted the separation width and used it for the threshold of separation width calculation. We repeated this procedure using Hermann grids with squares of different side-lengths. Separation width was incremented in steps of $5 \times 10^{\wedge}(-4)$ radians. The time interval between two steps was about 15 seconds.
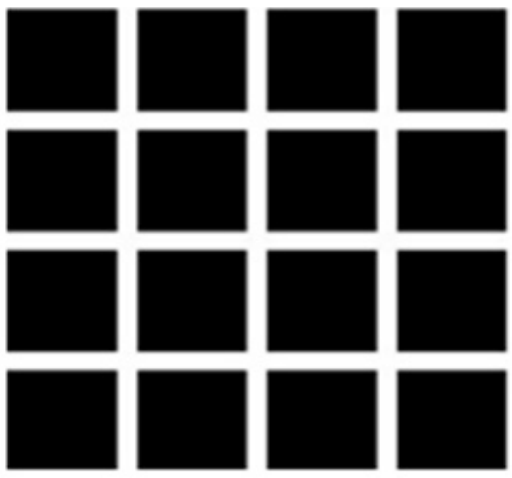

Figure 5a: The Hermann grid stimulus with small separation width. Illusory blobs are prominently visible at the intersections. 

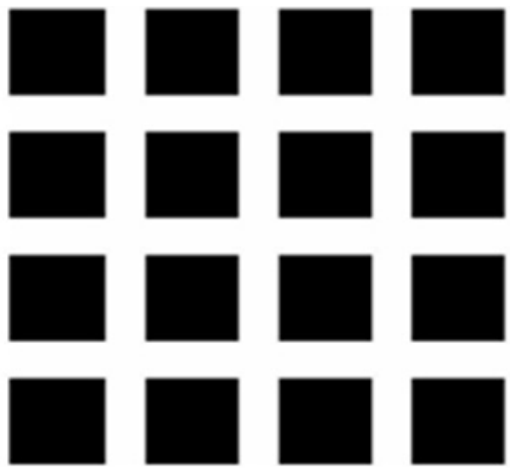

Figure 5b: The Hermann grid stimulus with medium separation width. Illusory blobs can be seen at the intersections, but are less prominent than Figure $5 \mathrm{a} .$.
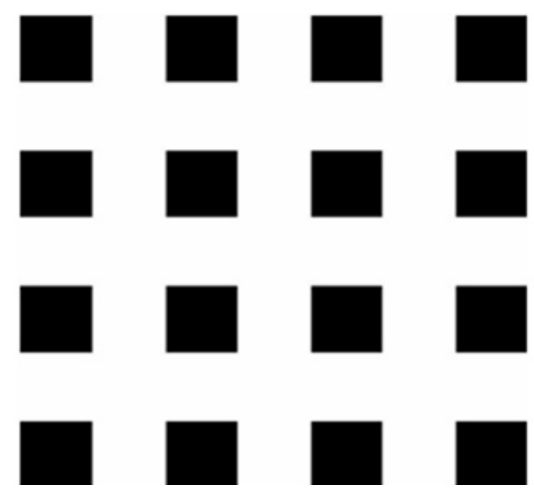

Figure 5c: The Hermann grid stimulus with large separation width. Illusory blobs become even less prominent.

\section{Observations and discussions}

Unlike Experiment 1 or 2, in this experiment two thresholds were involved. It was observed, that below a minimum separation between squares, the illusory effect of Hermann grid was not perceivable. We termed this threshold as the lower threshold' of separation. As its complement, a 'higher threshold' also exists, above which, the illusion cannot be seen.

From the experimental results, as illustrated in Figure $6 \mathrm{a}$ and $6 \mathrm{~b}$, we can see that for any subject the value of lower threshold' remains almost constant as the square size in Hermann grid changes. But the 'higher threshold' increases almost linearly as the size of the squares increases in the grid.

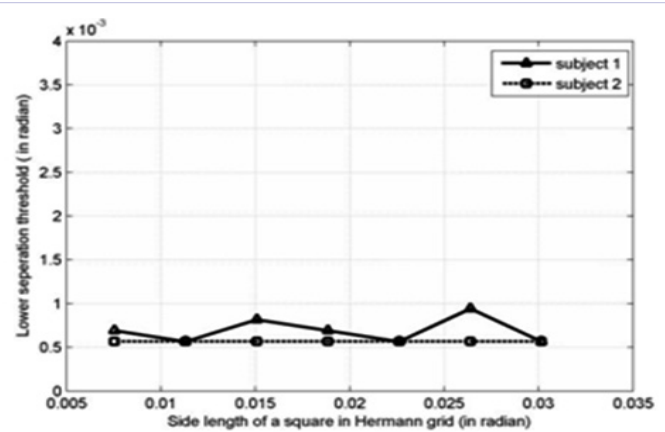

Figure 6a: Lower separation threshold vs. side length (of Hermann grid squares) graph..

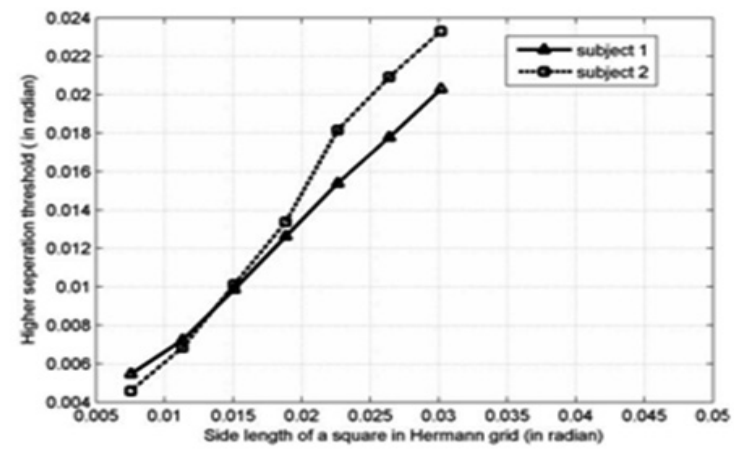

Figure 6b: Higher separation threshold vs. side length (of Hermann grid squares) graph.

\section{General Discussion and Inferences}

In this work the authors have designed and studied a series of psychophysical experiments on brightness contrast illusions. The 'detection of illusory effect' was the principal element in all the experiments. Based on the experimental results certain conclusions may be drawn. The first experiment appears different in its nature from the other two experiments. In the later two experiments we measured thresholds of some parameters which were related to the dimensions of the illusions while in the first we also have dealt with a parameter which was related to intensity values. However, we shall see from this general discussion that there happens to be interesting connectivity among all these with respect to the conclusions that we may draw.

From Experiment 2 we can see that when the gray-strip height is increased beyond a certain threshold, the illusory effect is no longer visible at the center of the gray-strip. This implies that the illusory effects in the Grating Induction illusion are confined to the boundaries of the gray-strip [11,12]. From Figure 4a and $4 \mathrm{~b}$, it can be seen that the visibility threshold of the illusory effect increases with the wavelength of the background sine/ square wave. This implies that the length scale of the illusory effect increases with the wavelength of the background waveform, a fact which suggests that the perceptual mechanism which is generating this illusion does not impose any inherent length scale of its own and the length scales observed in the illusory effect are derived from the input stimulus itself. The Oriented Difference of Gaussians (ODOG) spatial filter and its derivatives like LODOG or FLODOG of brightness perception are perceptual models based on this principle $[13,14]$. However, they too suffer from limitations. The White effect with a pretty long test patch demonstrates the failure of the ODOG type of models [15]. The White effect is a very interesting illusion, and it will not be a digression to discuss a little more about it context.

Like the Square Grating Induction illusion, the White effect illusion also consists of gray-strips over a square wave background. However, in this case we have two vertical gray-strips. One gray-strip lies between two black strips, whereas the other gray-strip lies between two white strips, as shown in Figure 7. It can be clearly seen that the first gray-strip looks darker than the second gray-strip. But unlike the Grating Induction illusion with horizontal gray-strips, the vertical gray-strips in this case appear 
to have uniform brightness i.e. the illusory effect is not confined only to the boundaries but visible throughout the gray-strips, and this happens irrespective of the width or height of the strips [15]. In other words the illusory effect has infinite length scale as opposed to the finite length scale for the Square Grating Induction illusion above. This is a fundamental difference between these two illusions, which otherwise superficially may seem to represent similar effects. Hence, these two are likely to have very different origins within the brain, much in the same way as White's illusion differs from the Simultaneous Brightness contrast illusion [16].

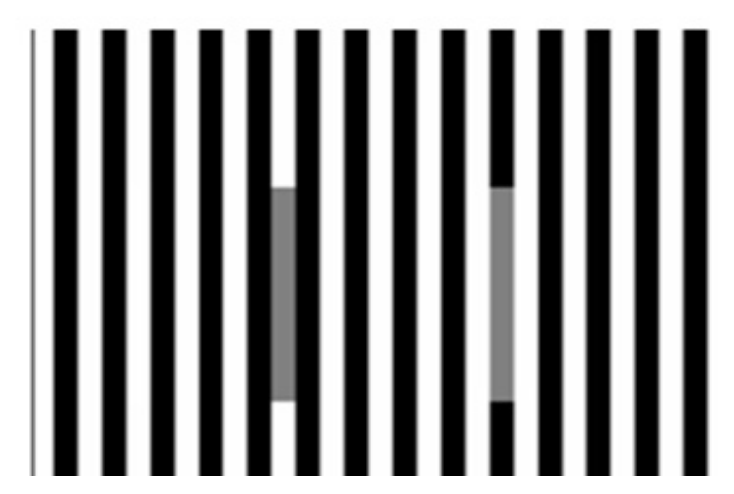

Figure 7: White's illusion. The gray-strips have the same intensity value but look dissimilar, but each of them seems to have homogeneous brightness all throughout.

In Experiment 3 we observe that both upper and lower thresholds are present in the Hermann grid illusion. While the lower threshold is independent of square-size, the upper threshold increases almost linearly with increasing square-size, i.e. it scales almost proportionally with square-size. This once again is a type of scaling behavior as seen in Experiment 2 above. This proportional variation of illusion size with stimulus size implies once again that the mechanism within the visual system that is generating this illusion is capable of generating illusions of all length scales, from very small to very large, and imposes no internal length scale of its own. Any filtering model of brightness illusion will find it hard to reproduce such a wide range of length scales, since most image filters will have typical length scales (e.g. sigma values of Gaussians and the filter sizes) of their own and would not respond for stimuli much smaller or much larger than that typical length scale. This is actually the reason why the ODOG type of models, despite being highly successful in explaining many brightness perception phenomena, have completely failed to explain certain such situations involving larger length scales [15]. The design of perceptual models of visual illusions should therefore take such scaling behavior into consideration. Such linear scaling behavior has also been observed previously for Mach Bands [17]. Several researchers are also attempting to solve the problem of scale integration from other perspectives [18,19].

Experiment 1, on the other hand, is quite a remarkable and novel one that shows us that even small changes to the shape of the grid squares are sufficient to destroy the illusory effect even without distorting the straightness of the larger squares themselves. This, on one hand, is in contradiction to what Geier et al. proposed, while on the other this implies that the visual mechanism producing this illusion can be very sensitive to the finer structure (i.e. the smaller length scales) of the stimulus [20]. This observation, it is easy to see, would also be difficult for the image filtering models to reproduce, including the highly successful ODOG model of Blakeslee \& McCourt and its derivatives, since in these models tiny changes in input will only produce tiny changes in output and will not wipe out the illusion altogether $[13,14]$.

We can thus infer from our experiments that any model of brightness perception should impose no length scale of its own, i.e. it should be scale invariant. At the same it must be sensitive to all the length-scales present in the input stimulus (as the structure of the stimulus at any length scale can entirely wipe out the illusory effect) and must derive all relevant length-scales from the input stimulus itself.

Thus our psychophysical studies of these brightness illusions have helped us to appreciate several visual phenomena and to draw some conclusions about the human visual system. We hope these observations will be of help in future to develop better models of visual perception.

\section{Acknowledgement}

Ashish Bakshi and Kuntal Ghosh would like to thank the TAC-DCSW, CCSD, Indian Statistical Institute, for providing a grant for the project 'Computational Models of Brightness Perception in Images' towards carrying out the present research work.

\section{References}

1. Eagleman DM. Visual illusions and neurobiology. Nature Reviews Neuroscience. 2001:2;920-926. doi:10.1038/35104092

2. Ehrenstein $\mathrm{WH}$ and Ehrenstein A. Psychophysical methods. In: Windhorst, U. and Johansson, H. (Eds.). Modern Techniques in Neuroscience Research, Springer Berlin Heidelberg, 1999;12111241. doi: 10.1007/978-3-642-58552-4_43

3. Gescheider GA. Psychophysics: the Fundamentals. New York: Psychology Press. 1999.

4. Spillmann L. The Hermann grid illusion: a tool for studying human perspective field organization. Perception. 1994;23(6):691-708. doi: $10.1068 / \mathrm{p} 230691$

5. Kien TT, Ren LJ, Maul T, Bargiela A. Outer Plexiform Layer Receptive Fields as Underlying Factors of the Hermann Grid Illusion. Proceedings of IEEE EMBS International Conference on Biomedical Engineering and Sciences. 2012:34-39. doi: 10.1109/ IECBES.2012.6498072

6. Davies NP, Morland AB. The Hermann-Hering grid illusion demonstrates disruption of lateral inhibition processing in diabetes mellitus. British Journal of Opthalmology. 2002;86(2):203-208.

7. Gilchrist JM, Pierscionek BK, Mann WM. Use of the Hermann grid illusion in the measurement of contrast perception in dyslexia. Vision Research. 2005;45(1):1-8.

8. McCourt ME. A spatial frequency dependent grating-induction effect. Vision Res. 1982;22(1):119-134. 
9. Schiller PH, Carvey CE. The Hermann grid illusion revisited. Perception. 2005;34(11):1375-1397.

10. McCourt ME, Foley JM. Spatial frequency interference on gratinginduction. Vision Res. 1985;25(10):1507-1518.

11. Land EH, McCann JJ. Lightness and retinex theory. J Opt Soc Am. 1971;61(1):1-11.

12. Rudd ME, Zemach IK. Quantitative properties of achromatic color induction: An edge integration analysis. Vision Res. 2004;44(10):971-981.

13. Blakeslee B, McCourt ME. A multiscale spatial filtering account of the White effect, simultaneous brightness contrast and grating induction. Vision Res. 1999;39(26):4361-4377.

14. Robinson AE, Hammon PS, de Sa VR. Explaining brightness illusions using spatial filtering and local response normalization. Vision Res. 2007;47(12):1631-1644.

15. Bakshi A, Roy S, Mallick A, Ghosh K. Limitations of the Oriented Difference of Gaussian Filter in Special Cases of Brightness Perception Illusions. Perception. 2016;45(3):328-336. doi: $10.1177 / 0301006615602621$
16. Ghosh K. A Possible Role and Basis of Visual Pathway Selection in Brightness Induction. Seeing Perceiving. 2012;25(2):179-212. doi: 10.1163/187847612X629946

17. Bakshi A, Ghosh K. Some insights into why the perception of Mach bands is strong for luminance ramps and weak or vanishing for luminance steps. Perception. 2012;41(11):1403-1408.

18. Rudd ME, Zemach IK. The highest luminance rule in achromatic color perception: Some counter examples and an alternative theory. J Vis. 2005;5(11):983-1003.

19. Kingdom FA. Lightness, brightness and transparency: A quarter century of new ideas, captivating demonstrations and unrelenting controversy. Vision Res. 2011;51(7):652-673. doi: 10.1016/j. visres.2010.09.012

20. Geier J, Bernath L, Hudak M, Sera L. Straightness as the main factor of the Hermann grid illusion. Perception. 2008;37(5):651-665. 